\title{
DETERMINATION BY X-RAY ABSORPTION OF REDOX INDUCED STRUCTURAL CHANGES IN IRON-SULFUR CLUSTER $F_{x}$ IN PHOTOSYSTEM I
}

I. SAGI

Department of Structural Biology, The Weizmann Institute of Science, Rehovot, Israel G. BUNKER

Department of Physics, Illinois Institute of Technology, Chicago, Illinois 60616, USA Y. Hochman, C. Carmeli* and M.T. Zeng

Department of Biochemistry, Tel Aviv University, Ramat Aviv, Tel Aviv 69978, Israel

X-ray absorption spectra of photosystem I core protein containing $F_{x}$, the low potential iron-sulfur cluster was measured. X-ray absorption of the iron $K$-edge was obtained in the reduced and oxidized forms of $\mathrm{F}_{\mathrm{x}}$. An edge shift of $0.5 \mathrm{eV}$ to lower energy and a change in the X-ray absorption near edge structure was observed. Some of the alterations in edge shape of $F_{x}$ are interpreted to indicate structural changes of the iron-sulfur cluster in $F_{x}$ upon reduction.

PACS numbers: 78.70.Dm

\section{Introduction}

Photosystem I (PSI) is a membrane-bound pigment-protein complex which mediates electron transfer from reduced plastocyanine to ferredoxin. Five different electron-transfer intermediates, namely $A_{0}, A_{1}, F_{x}, F_{A}$, and $F_{B}$, are known to be involved in electron transfer from P700 to ferredoxin. $A_{0}$ and $A_{1}$ are believed to be a monomeric chlorophyll and a phylloquinone respectively, whereas $\mathrm{F}_{\mathbf{x}}, \mathrm{F}_{\mathbf{A}}$, and $F_{B}$ are all iron-sulfur [4Fe-4S] clusters [1]. $F_{X}$ is well adapted to its role as an early electron mediator in PSI located between the phylloquinone and the $F_{A} / F_{B}$ clusters as verified by low resolution analysis of PSI crystals [2]. Various proteins which contain related clusters may have large differences in their characteristic midpoint potentials [3]. The differences were suggested to be mostly due to the degree of electrostatic interaction of the cluster through hydrogen bonds of amides and the water molecules in the protein environment [4].

${ }^{*}$ Corresponding author. 
Yet $F_{\mathbf{x}}$, which has one of the lowest known midpoint potentials of iron-sulfur clusters, is in membrane and might be expected to have higher potential. It is suggested therefore that structural changes during reduction might play an important role in this protein. An increase of up to $0.09 \AA$ in the $\mathrm{Fe}-\mathrm{S}$ bond length was observed on the reduction of various relevant iron-sulfur clusters $[5,6]$. These findings suggested that reversible rearrangement in structure might be a mechanism for stabilization of intermediate states and for rapid mediation of electrons.

\section{Experimental}

PSI complex was prepared from thylakoid isolated from the cyanobacteria Synechocystis sp. Strain PCC 6803 , by triton $\mathrm{x}-100$ solubilization. It was first purified on a DEAE-cellulose column followed by sucrose gradient centrifugation [7]. The minor subunits were then dissociated by $2.0 \mathrm{M} \mathrm{NaI}$ [1] and the PSI core subunits then were purified on sucrose gradient centrifugation. The PSI core protein, containing $2 \mathrm{mg} / \mathrm{ml}$ chlorophyll $(0.25 \mathrm{mM} \mathrm{Fe})$ and $50 \%$ glycerol, was applied to a $20 \mu \mathrm{l}$ sample holder and quickly frozen in liquid nitrogen for X-ray measurements. The sample holder was made out of $0.5 \mathrm{~mm}$ thick copper with $2 \mathrm{~mm} \times 20 \mathrm{~mm}$ windows sealed by $0.05 \mathrm{~mm}$ thick mylar. $\mathrm{F}_{\mathrm{x}}$ in the reduced form was pre-reduced with $10 \mu \mathrm{M}$ phenazine methosulfate and dithionite at pH 10 in the presence of light prior to freezing.

Flash induced transient absorption changes in P700 was measured using a modified flash photolysis setup [8] which included a $10 \mathrm{~ns}$ flash from Quantal $\mathrm{Nd}-Y A G$ laser at $532 \mathrm{~nm}$, a measuring beam from a diode laser $820 \mathrm{~nm}$. The absorption changes were monitored by photodiodes, amplified by amplifier, interfaced by Tektronix TDS 520A Digitizing Oscilloscope and recorded on a PC computer. The reaction medium contained $50 \mathrm{mM}$ tris, $\mathrm{pH} 8.0,1.7 \mathrm{mM}$ sodium ascorbate, $1.7 \mu \mathrm{M}$ dichlorophenol indophenol and $30 \mu \mathrm{g} / \mathrm{ml}$ chlorophyll.

$\mathrm{X}$-ray absorption fluorescence data were collected at the National Synchrotron Light Source, Brookhaven National Laboratory, on beam line X-9A using a double flat $\mathrm{Si}(111)$ crystal monochromator. X-ray fluorescence from iron $\left(K_{\alpha}\right.$-peak) was collected using an energy germanium $(\mathrm{Ge})$ detector. All experiments were carried out at $30 \mathrm{~K}$. X-ray absorption data having $3 \mathrm{eV}$ resolution were recorded by counting at specific energy for 3 seconds and incrementing the energy by $0.5 \mathrm{eV}$ from $50 \mathrm{eV}$ below the edge to $100 \mathrm{eV}$ above the edge. An iron $\mu \mathrm{m}$ foil transmission spectrum was measured simultaneously and used as a calibration standard to account for any shifts in the monochromator. X-ray absorption edge data were manipulated and analyzed using standard procedures $[9,10]$.

\section{Results and discussion}

To study the function of PSI at physiological temperature flash-induced transient absorption changes of P700 at $820 \mathrm{~nm}$ were measured. The time constants derived from the kinetic transients were used to identify the relative contributions of various electron acceptors to P700. The lifetimes of back electron transfer from different components of the PSI acceptor side to $\mathrm{P} 700^{+}$range from $10 \mathrm{~ns}$ $\left(\mathrm{A}_{0}\right) ; 10 \mu \mathrm{s}\left(\mathrm{A}_{1}\right) ; 0.8 \mathrm{~ms}\left(\mathrm{~F}_{\mathrm{x}}\right)$; and $50 \mathrm{~ms}\left(\mathrm{~F}_{\mathrm{A}} / \mathrm{F}_{\mathrm{B}}\right)$ [1]. Figure $1 \mathrm{~A}$ showed that in PSI complex, the flash induced a rapid unresolved increase in absorption at $820 \mathrm{~nm}$ 


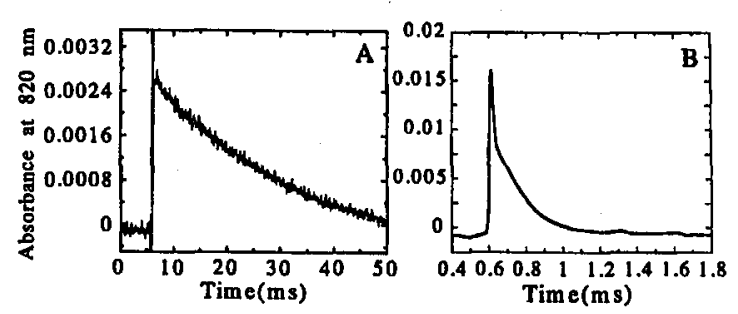

Fig. 1. Measurement of the rate of P700 reduction following its oxidation by $10 \mathrm{~ns}$ Nd-YAG laser flash in PSI complex and PSI core protein from Synechocystis. (A) In PSI complex. (B) In PSI core protein.

that was typical of P700 oxidation which is followed by a decay with half time of $30 \mathrm{~ms}$. This decay was due to $\mathrm{P} 700^{+} \mathrm{F}_{\mathrm{A}}-/ \mathrm{F}_{\mathrm{B}}-$ recombination. In contrast, the measurement with PSI core protein showed that the major component, with half lifetime of $0.5 \mathrm{~ms}$, was described to a back reaction from $\mathrm{F}_{\mathrm{x}^{-}}$to $\mathrm{P} 700^{+}$(Fig. 1B). It indicated that $\mathrm{F}_{\mathrm{x}}$ was not disintegrated in the preparation since in its absence an approximately $10 \mu$ s decay caused by the recombination of $\mathrm{P}^{2} 00^{+} \mathrm{A}_{1}$ - takes place. It also confirmed that iron-sulfur clusters $\mathrm{F}_{\mathrm{A}}$ and $\mathrm{F}_{\mathrm{B}}$ were removed functionally from PSI core preparation. The existence of functional $\mathrm{F}_{\mathrm{X}}$ in this preparation is confirmed by EPR measurements at $10 \mathrm{~K}$. Following photoreduction at $293 \mathrm{~K} \mathrm{EPR}$ resonance were observed at $8 \mathrm{~K}$ at $g=2.0025$ and $g=1.88,1.78$ due to $\mathrm{P} 700^{+}$and $\mathrm{F}_{\mathrm{X}^{-}}$respectively (data not shown). This ensured that the measurement for $\mathrm{X}$-ray absorption of the iron in $\mathbf{F}_{\mathbf{x}}$ of PSI would not be interfered by other iron-sulfur clusters $\mathrm{F}_{\mathrm{A}} / \mathrm{F}_{\mathrm{B}}$ in this protein complex.

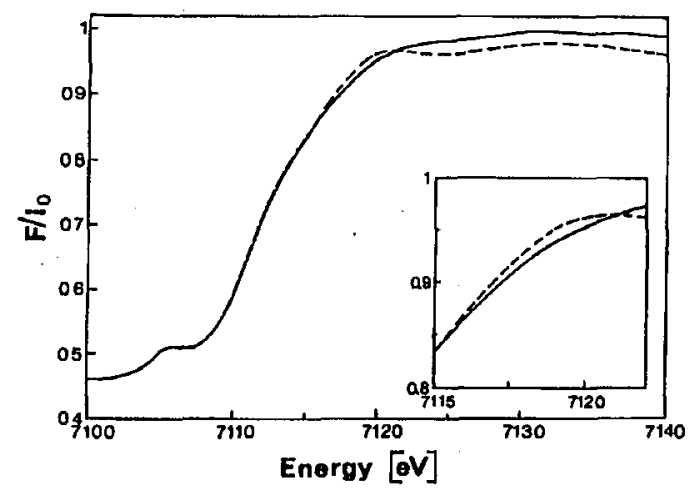

Fig. 2. X-ray absorption of the $K$-edge of iron in the oxidized (solid) and reduced (dashed) states of the $[4 \mathrm{Fe}-4 \mathrm{~S}]$ iron-sulfur cluster $\mathrm{F}_{\mathbf{x}}$ in PSI core protein.

Figure 2 shows the X-ray fluorescence data of the oxidized (solid) and reduced (dashed) forms of PSI core protein. The two main pre-edge transitions, $1 s-3 d$ at $7107 \mathrm{eV}$ and $1 s-4 p$ at $7118 \mathrm{eV}$, of both oxidation states are very similar and consistent with other iron-sulfur proteins and relevant model compound data [6]. 
The reduced $F_{x}$ spectrum is shifted by $0.5 \mathrm{eV}$ to lower energy (Fig. 2, inset), as expected, upon reduction [6]. The unique characteristic which can be detected is a significant difference in the edge shape (in the region of 7115-7130 eV). This region designated as the X-ray absorption near edge structure (XANES) region, contains detailed and accurate structural information of the first shell surrounding the photo-absorbing atom $[11,12]$. The second derivative of the $K$-edge spectra, shown in Fig. 3, emphasizes the changes in the XANES region between the oxidized and reduced states of $F_{\mathbf{x}}$. Interpretation of XANES data was known to be difficult

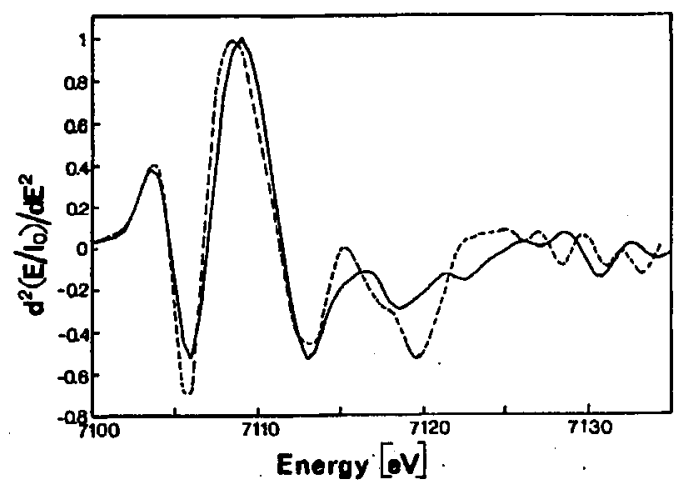

Fig. 3. Second derivative spectra of $F_{x}$ in its oxidized (solid) and reduced (dashed) state. Second derivatives were generated by fitting regions to third-order polynomials and computing the second derivative analytically.

due to the need to account for multiple scattering corrections in this region. The recent advances in calculating the XANES spectra, which explicitly account for all types of multiple scattering processes, stimulated the interest in this informative domain [12]. A similar trend of alteration in XANES spectra upon reduction of iron-sulfur proteins is observed in rubredoxin and related model compounds reported. A consistent shift of $0.7 \mathrm{eV}$ to lower energy and a great similarity of the pre-edge transitions and the XANES region can be detected [5].

The crystal structures of the oxidized and reduced forms of rubredoxin from Pyrococcus furiosus (Rdpf) provided an accurate (1.8 $\AA$ resolution) and detailed comparison of the two states [13]. The main differences between the structure of the oxidized and reduced forms of Rdpf are an increase in the iron-sulfur bond lengths by an average of $0.04 \AA$ and a decrease in the NH-S hydrogen bond distances by $0.09 \AA$ following reduction of the oxidized form. It has been speculated that reduction of the hydrogen bond network around the iron cluster can help to stabilize the negative charge introduced by reduction. A similar trend of an increase in bond length of the iron-iron and iron-sulfur bond, respectively was observed on reduction in crystals of HIPIP (high potential iron proteins) and various ferredoxins [14]. These results confirmed our observation which indicated that X-ray absorption changes could be observed on reduction of $F_{x}$ and hence structural changes took place. 
Calculation of the XANES spectra of the oxidized/reduced forms of $F_{x}$ and an extensive analysis of relevant model compounds can provide a direct structural and functional relations in $F_{x}$ of PSI. This approach will lead us to a better understanding of the $F_{x}$ iron-sulfur cluster which has a central role in the stabilization of electrons coming from the special pair chlorophylls which make up the P700 reaction center of PSI.

\section{References}

[1] J.H. Goldbeck, D.A. Bryant, Current Topics in Bioenerg. 16, 83 (1991).

[2] N. Krauss, W. Hinrichs, I. Witt, P. Fromme, W. Pritzkow, Z. Dauter, C. Betzel, K.S. Wilson, K.H. Witt, W. Saenger, Nature 361, 326 (1993).

[3] R. Cammack, in: Iron-Sulfur Protein Research, Eds. H. Matsubara, Y. Katsube, K. Wada, Springer-Verlag, Berlin 1986, p. 40.

[4] R. Langen, G.M. Jensen, U. Jacob, P.J. Stephens, A. Warshel, J. Biol. Chem. 267, 25625 (1992).

[5] B.K. Teo, R.G. Shulman, G.S. Brown, A.E. Meixner, J. Am. Chem. Soc. 101, 5624 (1979).

[6] R.G. Shulman, P. Eisenberger, B.K. Teo, B.M. Kincaid, G.S. Brown, J. Mol. Biol. 124, 305 (1978).

[7] R. Nechushtai, P. Muster, A. Binder, V. Liveanu, N. Nelson, Proc. Natl. Acad. Sci. USA 80, 1179 (1983).

[8] C. Carmeli, M. Gutman, Fed. Eur. Biochem. Soc. Lett. 141, 88 (1982).

[9] E.A. Stern, S.M. Heald, in: Handbook on Synchroton Radiation, Ed. E. Kok, North-Holland, Amsterdam 1983, Ch. 10, p. 955.

[10] C. Carmeli, J.Y. Huang, D.M. Mills, A.T. Jagendorf, A. Lewis, J. Biol. Chem. 261, 16969 (1986).

[11] G. Bunker, E.A. Stern, Phys. Rev. Lett. 52, 1990 (1984).

[12] J.J. Rehr, J. Mustr de Leon, S.I. Zabinsky, R.C. Alberts, J. Am. Chem. Soc. 113, 5135 (1991).

[13] M.W. Day, B.T. Hsu, L. Joshua-Tor, J. Park, Z. Hao, M.W. Adams, D.C. Rees, Protein Science 1, 1494 (1992).

[14] C.W. Carter, Proc. Natl. Acad. Sci. USA 69, 3526 (1972). 